\title{
EYE MOVEMENT PATTERNS AND DRIVING PERFORMANCE
}

\author{
Zheng Bian, Russell Pierce, \& George Andersen \\ Department of Psychology, University of California, Riverside \\ Riverside, California, USA \\ Email: andersen@ucr.edu
}

\begin{abstract}
Summary: In the current study we examined the relationship between drivers' eye movement patterns and driving performance in a dual-task driving paradigm. Drivers performed two tasks in a driving simulator. In a car following task, drivers were asked to maintain a constant headway from a leading vehicle as it varied its speed. In the second task (light detection task), drivers tried to detect changes in peripheral traffic lights. The performance in the car following task was measured with headway distance and RMS, and the performance in the light detection task was measured with response time and accuracy. We found that the frequency of fixations, fixation duration and spatial distribution of fixations were significantly correlated with drivers' performance in the driving tasks. Specifically, driving performance improved with fewer eye movements, longer fixation durations and smaller spatial distribution of fixations.
\end{abstract}

\section{INTRODUCTION}

Driving is a complex task that involves performing multiple visual tasks either concurrently or within very short temporal ranges. These tasks include steering control (Wallis, Chatziastros, Tresilian \& Tomasevic, 2007), braking (Fajen, 2005), car following (Ni, Kang \& Andersen, 2010), and collision detection (Ni \& Andersen, 2008). Previous studies have shown that observers' ability to attend and process information in a visual scene is very limited (Rensink, 2002). Failing to attend to critical information in a driving context could result in increased risk of a vehicle crash. An important issue in driving research is to determine what characteristics of drivers are associated with potential crash risk.

Previous research has shown that the Useful Field of View (UFOV), the area of visual field within which information is processed without an eye movement, can predict crash risk for older drivers (Owsley, Ball, McGwin, Sloane \& Roneker, 1998). However, UFOV is measured using a 2D display and does not assess visual processing of information that varies in depth and is present when driving in a 3D world. Previous research has shown that the extent of spatial attention is limited along the depth axis (Andersen, 1990). In addition, research has shown that drivers' ability to detect important targets during driving (detecting changes in traffic lights) varied as a function of distance from the driver (Andersen, Ni, Bian and Kang, in press). These results can not be accounted for by UFOV that only assesses the limitations of spatial attention of a driver in the horizontal and vertical dimensions.

A related line of research that is important in understanding attention and visual processing during driving involves measuring the eye movement patterns of drivers. For instance, Crundall and Underwood (1998) compared the scanning patterns of experienced and novice drivers. They found that, for experienced drivers, the standard deviation of fixation was greater in both 
horizontal and vertical dimensions when driving on a two lane road than on rural and suburban roadways. Novice drivers, on the other hand, did not show differential scanning patterns according to the type of roadway. Carter and Laya (1998) found that experienced drivers, as compared to novice drivers, had longer fixation durations while the standard deviation of fixations did not differ between the two groups. Crundall, Shenton and Underwood (2004) found that during intentional car following, drivers had longer fixation durations and a smaller range of fixations. Other studies have found that the presence of mental tasks lead to a decrease in the standard deviation of gaze position (Recarte \& Nunes, 2000, 2003; Victor, Harbluk \& Engstrom, 2005). These results, considered together, suggest an important relationship between eye movement patterns and driving performance.

In the current study, we examined whether scanning patterns could predict driving performance in a dual-task driving paradigm. College age drivers were asked to maintain a constant distance from a lead vehicle as it varied its speed. In addition, they had to respond to traffic light changes that varied in eccentricity (horizontal and vertical dimensions) as well as distance. Drivers' eye movements were recorded and analyzed in addition to performance in the driving tasks.

\section{EXPERIMENT}

\section{Methods}

Drivers. The drivers were 21 undergraduate students (9 males and 12 females, mean age $=21.6 \pm$ 2.1) who were paid for their participation. All drivers were screened using several perceptual and cognitive tests, including Snellen acuity, contrast sensitivity, forward and backward digit span, perceptual encoding, Useful Field of View, and the Kaufman Brief Intelligence Test. All drivers had normal or corrected-to-normal vision, had a minimum of 2 years of driving experience, and were naïve to the purpose of the study.

Apparatus. A Dell 670 Workstation desktop computer was used to present the displays on a 23 in. flat screen LCD monitor with a pixel resolution of $1024 \times 768$ and a refresh rate of $30 \mathrm{~Hz}$. The viewing distance was $91.5 \mathrm{~cm}$ (36 in.), and the visual angle was $34.7^{\circ} \times 25.8^{\circ}$. A Thrustmaster Formula T2 steering system (with acceleration and brake pedals) was used for closed-loop control of the simulator. The foot pedal and a BG systems serial box (analog to digital converter system) were used to produce closed loop control that was updated at $36 \mathrm{~Hz}$. Eye movements were recorded with an Eyelink II eye tracker (SR Research) at a sampling rate of $500 \mathrm{~Hz}$.

Stimuli. The stimuli were computer generated 3-D scenes composed of a one-way road with three lanes, a leading vehicle (LV), and buildings on both sides of the road (see Figure 1). The lanes width was 3.8m, demarked by dashed lines ( 2 meters in length positioned every 2 meters along the roadway). Asphalt was simulated using black and white gravel texture pattern. The driver and the LV were located in the center lane. A silver colored sedan was used to represent the LV subtending a horizontal visual angle of $5.5^{\circ}$ at a headway distance of $20.5 \mathrm{~m}$. Digital photographs of real buildings and vehicles were used as texture maps for the roadway scenes. The images were digitally altered to increase the realism of the simulator scene (e.g., remove specular highlights, add shading) and were scaled to be appropriate for the 3D geometry of the simulation. The average luminance of the driving scene was $24.7 \mathrm{~cd} / \mathrm{m}^{2}$. 


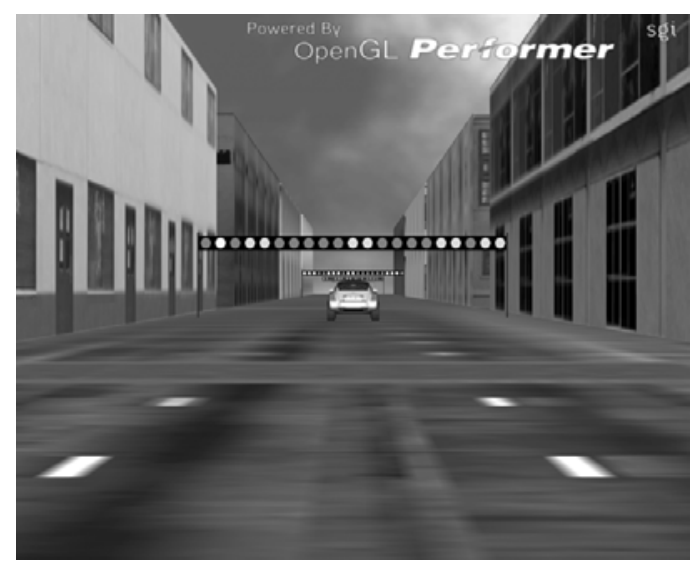

Figure 1. Static image of driving scene used in the present study

In the car following task, the LV's average speed was $60 \mathrm{kmph}$ (37.3 mph). It varied its velocity according to a sum of three equal energy sinusoids (i.e. each sine wave's peak accelerations and decelerations were equivalent). The amplitudes for the three sine waves were 9.722, 3.889, and $2.778 \mathrm{kmph}$, respectively. The average range of speed produced by the sum of sinusoidal functions was $\pm 14.3 \mathrm{kmph}$ about the mean speed.

In the light detection task, a light array with 21 lights was simulated, each of which randomly presented in red or green color. The simulated size of the light array was $12.68 \mathrm{~m} \times 0.60 \mathrm{~m}$, and it was placed at $2.7 \mathrm{~m}$ above the roadway. Each light had a diameter of $0.5 \mathrm{~m}$. The potential targets were the $3^{\text {rd }}$, the $6^{\text {th }}$, and the $9^{\text {th }}$ to either side from the center.

Design. In the light detection task, three independent variables were examined: (1) the distance of the light change (24, 36, 48, or $72 \mathrm{~m})$, (2) the side of the target (the light that changes color) on the light array (left vs. right), and (3) the horizontal position of the target on the light array relative to the center $\left(3^{\text {rd }}, 6^{\text {th }}\right.$, or $9^{\text {th }}$ position). All variables were run as within-subject variables.

Procedure. The experiment was run in a darkened room. Each trial started with a preview phase with both the LV and the drivers' vehicle moving at a constant speed of $60 \mathrm{kmph}$ with a constant separation of $20.5 \mathrm{~m}$. The drivers were instructed to remember this distance as the desired headway distance for the rest of the trial. After 5 seconds, drivers heard a tone which indicated that the control input from the drivers was allowed. The LV varied its speed according to the sum of three sine wave frequencies and the drivers were instructed to maintain the pre-determined headway distance by using the acceleration and brake pedals. Feedback for the car following task was used by activating a horn sound if the distance headway (the distance between the drivers' vehicle and the LV) exceeded $27.3 \mathrm{~m}$.

While driving down the roadway the drivers would pass a light array every 75 meters with 21 red or green colored lights. Each array had a different random order of red and green lights. When the drivers' vehicle was $24,36,48$, or 72 meters away from the light array, one of the lights would change its color to yellow. The target was the $3^{\text {rd }}, 6^{\text {th }}$, or $9^{\text {th }}$ light located on either side from the center. The drivers were instructed to indicate whether the target was on the left or the right hand side of the light array as soon and as accurately as possible. They made a response by pressing the left button or the right button on the steering wheel with their index fingers. The 
feedback of the light detection task was given by activating either a high-tone sound (indicating a correct response) or a low-tone sound (indicating a wrong response). If the drivers failed to make a response before passing the light array, a neutral sound was activated as a reminder to the drivers. On each trial, each of the 24 combinations of distance, side, and target position occurred once, resulting in 24 light detection responses. The order of the condition was randomized.

The experiment contained 3 blocks. The first block was a practice block that allowed drivers to become familiar with the control dynamics of the simulator and the driving tasks. The practice block was followed by two experimental blocks. There were 3 trials in each block and 24 light arrays in each trial. The drivers were instructed to perform both tasks equally well. A break was given after each block. Each trial lasted for approximately 105 seconds and the duration of the whole experiment was about 45 minutes.

\section{Results}

Car Following Task. For the car following task we examined the average headway distance and RMS velocity error. The headway distance measures the average following distance between the drivers' vehicle and the LV. The RMS error measures the overall error in matching the LV's speed. On average, drivers were following the $\mathrm{LV}$ at a distance of $16.57 \pm 2.69 \mathrm{~m}$, suggesting that drivers tended to follow at a closer distance than instructed $(20.5 \mathrm{~m})$. The RMS error was $5.44 \pm 1.0 \mathrm{kmph}$, suggesting that drivers were tracking the LV’s speed with relatively high accuracy.

Light Detection Task. For the light detection task, we examined accuracy and response time. A 4 (distance) $\times 2$ (side of the target) $\times 3$ (horizontal position of the target) ANOVA with repeated measures were conducted for both accuracy rate and response time. For accuracy, there was a significant main effect of target distance $(F(3,60)=17.93, p<.01)$, a significant main effect of target position on the banner $(F(2,40)=3.87, p<.05)$, and a significant interaction between these two variables $(F(6,120)=3.22, p<.01)$. According to this result, the average accuracy was close to $100 \%$ when the target distance was $36 \mathrm{~m}, 48 \mathrm{~m}$, and $60 \mathrm{~m}$. When the light change occurred at $24 \mathrm{~m}$ away from the drivers, the average accuracy decreased from $97 \%$ to $94 \%$ as the target moved further from the center.

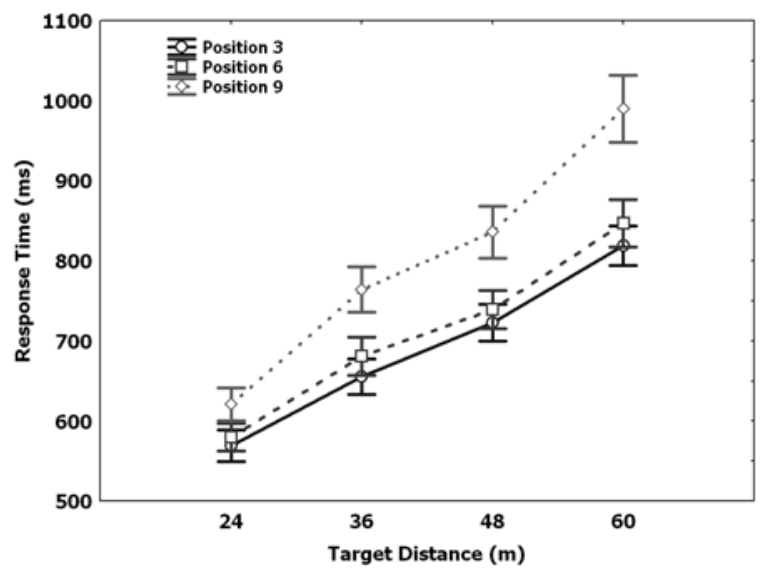

Figure 2. Response time to light changes as a function of target distance and horizontal position of target (Error bars represent \pm 1 standard error) 
For response time, there was a significant main effect of target distance $(F(3,60)=128.03, p$ $<.01)$, target position on the banner $(F(2,40)=34.68, p<.01)$, and a significant interaction between these two variables $(F(6,120)=3.11, p<.01)$. According to this result, the response time increased as a function of target distance and target position on the banner. When the target was furthest from the center of display, the increase of response time with increased target distance was larger than when the target was closer to the center of display (see Figure 2).

Eye movements. For each driver, we measured the frequency of eye movement, average duration per fixation, and the spatial distribution of fixations. On average, drivers were making $2.50 \pm$ 0.71 fixations per second with $0.40 \pm 0.15$ seconds per fixation. The spatial distribution of fixations was measured using the standard deviation of drivers' gaze positions. On average, this value was $3.65 \pm 0.61$ degrees.

In order to examine whether eye movement patterns could predict driving performance, a Pearson correlation was conducted between the three eye movement measures and the driving performance measures for both tasks. For the car following task, we found a significant correlation between the headway distance and the fixation duration $(r=0.47, p<.05)$. None of the eye movement measures significantly predicted RMS $(p>.05)$. For the light detection task, none of the eye movement measures significantly predicted accuracy rate $(p>.05)$ probably due to the high accuracy rate for all drivers. The response time, however, could be predicted by the frequency of fixation $(r=0.65, p<.01)$, the fixation duration $(r=-.62, p<.01)$, and the spatial distribution of fixations $(r=0.62, p<.01$ ) (Figure 3 ). We also found that the three eye movement measures were significantly correlated between each other $(p<.05)$. These results suggest that drivers with a small spatial distribution of fixations tend to make fewer eye movements and longer fixations than drivers with a large spatial distribution of fixations. As a result, they could follow the LV at a closer distance and respond to traffic light changes faster. Figure 4 shows the results for a typical driver with a small distribution of fixations and for a driver with a large distribution of fixations. The driver on the left mainly focused his/her attention around the central area while driver on the right spread his/her fixations to both side of the driving scene. Their average response times were $637 \mathrm{~ms}$ and $862 \mathrm{~ms}$, respectively.

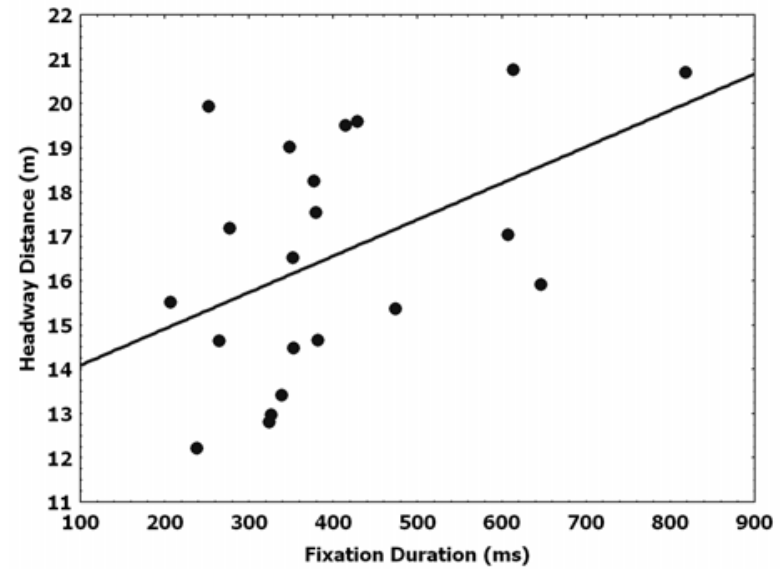

(a)



(b)

Figure 3. (a) correlation between fixation duration and headway distance; (b) correlation between fixation duration and response time 

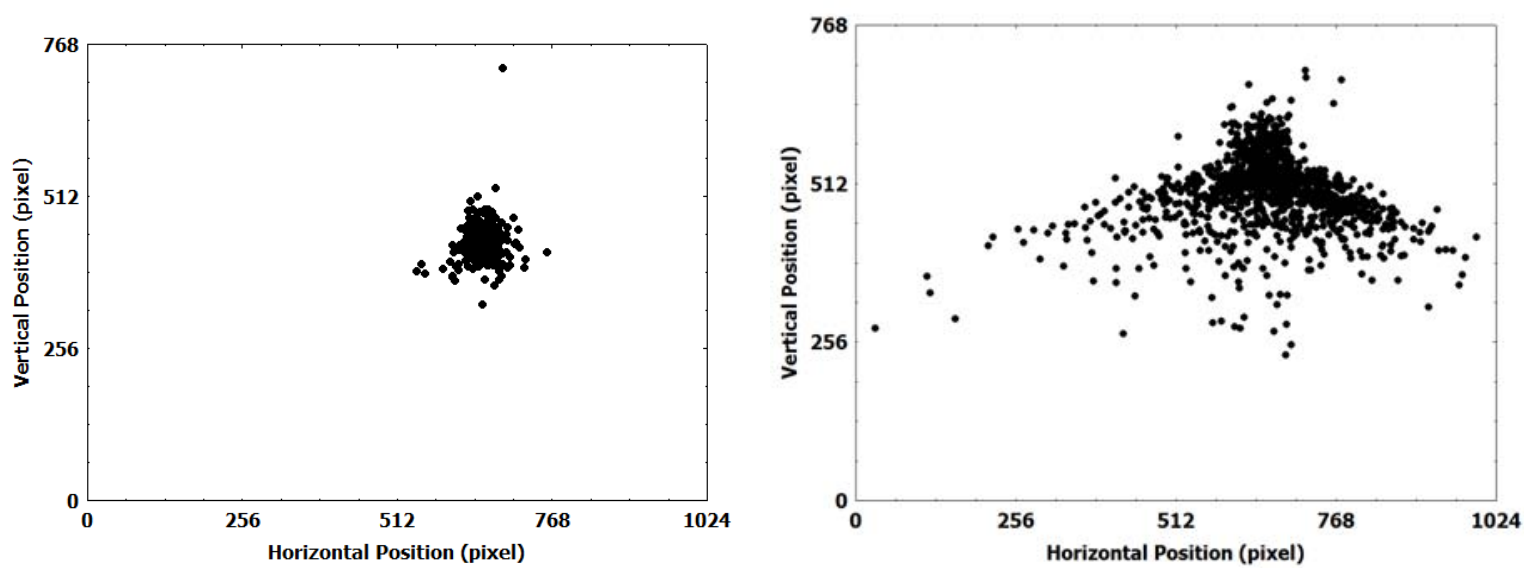

Figure 4. Location of eye fixations for (a) a driver with small spatial distribution of fixations and (b) a driver with large spatial distribution of fixations

We also examined whether this significant correlation between eye movement patterns and driving performance was mediated by UFOV. We found that UFOV (selective attention measure) was not significantly correlated with headway distance $(r=0.18, p=.44)$, RMS $(r=0.03, p$ $=.90)$, or response time $(r=0.01, p=.97)$. UFOV was not significantly correlated with any of the eye movement measures, either $(p>.05)$. This suggests that the eye movement patterns could predict performance in the current dual task driving paradigm independent of UFOV.

\section{DISCUSSION}

In this study, we examined whether eye movement patterns could predict performance in a dualtask driving paradigm. We found that both the headway distance in the car following task and the response time in the traffic light change detection task could be predicted by the eye movement patterns. Drivers who make less frequent fixations, fixate longer in a small spatial area respond faster to the traffic light changes than drivers who frequently make short fixations spreading a bigger area. Our results are consistent with previous studies showing longer fixation durations for experienced as compared to novice drivers (Carter \& Laya, 1998) and for trained as compared to untrained drivers (Seya \& Nakayasu, 2008). Our results could not be explained by UFOV, since UFOV measures did not correlate significantly with any of our driving performance measures or the eye movement patterns.

In the current study, the two tasks used were a car following task and traffic light change task. It is important to examine whether our finding extend to other tasks, such as steering control and detecting roadway hazards. Boot, Kramer, Becic, Weigmann and Kubose (2006) found that observers who made more fixations were less accurate in a dynamic search task. When instructed to make fewer eye movements, their performance improved to the same level as the observers who initially made fewer fixations. This suggests that scan strategy was important in detecting transient changes. One interesting topic for future research is to examine whether driving performance can improve by changing scan strategies. On a related issue, previous research found age-related decrement in performance in a car following task (Ni, Kang \& Andersen, 2010). Another topic for future research is to examine whether age-related decrement in driving tasks could be predicted by an age-related difference in eye movement patterns, and whether we could improve older drivers’ performance by training them to use a different scan strategy. 


\section{ACKNOWLEDGMENT}

This research was supported by NIH AG031941 and EY18334.

\section{REFERENCES}

Andersen, G. J., Ni, R., Bian, Z. \& Kang, J. J. (2011). Limits of spatial attention in threedimensional space and dual-task driving performance. Accident Analysis and Prevention, 43, $381-390$.

Andersen, G. J. (1990). Focused attention in three-dimensional space. Perception and Psychophysics, 47, $112-120$.

Boot, W. R., Kramer, A. F., Becic, E., Wiegmann, D. A., \& Kubose, T. (2006). Detecting transient changes in dynamic displays: the more you look, the less you see. Human Factors, 48 (4), 759 - 773.

Carter, C. J., \& Laya, O. (1998). Drivers' visual search in a field study and in a driving simulator. In A. G. Gale et al. (Eds), Vision in Vehicles VI, 21 - 31. Amsterdam: Elsevier.

Crundall, D. E. \& Underwood, G. (1998). Effects of experience and processing demands on visual information acquisition in drivers. Ergonomics, 41, 448 - 458.

Crundall, D. E., Shenton, C. \& Underwood, G. (2004). Eye movements during intentional car following. Perception, 33, 975 - 986.

Fajen, B. R. (2005). Callibration, information, and control strategies for braking to avoid a collision. Journal of Experimental Psychology: Human Perception \& Performance, 31, 480501.

Ni, R., Kang, J. J. \& Andersen, G. J. (2010). Age-related declines in car following performance under simulated fog conditions. Accident Analysis and Prevention, 42, 818 - 826.

Owsley, C., Ball, K., McGwin, G., Slaone, M. E., \& Ronker, D. L. (1998). Visual processing impairment and risk of motor vehicle crash among older drivers. Journal of the American Medical Association, 279, 1083-1088.

Recarte, M. A., \& Nunes, L. M. (2000). Effects of verbal and spatial-imagery tasks on eye fixations while driving. Journal of Experimental Psychology: Applied, 6(1), 31-43.

Recarte, M. A., \& Nunes, L. M. (2003). Mental workload while driving: Effects on visual search, discrimination, and decision making. Journal of Experimental Psychology: Applied, 9(2), 119-137.

Rensink, R. A. (2002). Change detection. Annual Review of Psychology, 129, 245-277.

Seya, Y., \& Nakayasu, H. (2008). Visual search of trained and untrained drivers in a driving simulator. Japanese Psychological Research, 4, 242 - 252.

Victor, T., Harbluk, J. L. \& Engstrom, J. A. (2005). Sensitivity of eye-movement measures to invehicle task difficulty. Transportion Research Part F. 8, 167 - 190.

Wallis, G., Chatziastros, A., Tresillian, J., \& Toasevic, N. (2007). The role of visual and nonvisual feedback in a vehicle steering task. Journal of Experimental Psychology: Human Perception \& Performance, 33, 1127-1144. 